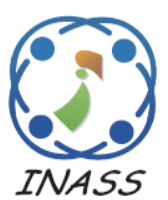

\title{
Intelligent Control of Power System Stabilizer Based on Archimedes Optimization Algorithm - Feed Forward Neural Network
}

\author{
Widi Aribowo $^{1 *} \quad$ Supari Muslim $^{1} \quad$ Bambang Suprianto $^{1}$ \\ Subuh Isnur Haryudo ${ }^{1} \quad$ Aditya Chandra Hermawan ${ }^{1}$ \\ ${ }^{1}$ Departement of Electrical Engineering, Universitas Negeri Surabaya, Indonesia \\ * Corresponding author's Email: widiaribowo@unesa.ac.id
}

\begin{abstract}
A power system is a collection of equipment that has characteristics. Power system stability is maintaining how the system can return to its position. Power system stabilizer (PSS) is a device used to maintain the stability of the power system due to load changes. The archimedes optimization algorithm (AOA) is a metaheuristic method based on the force that occurs in the fluid due to a load. The method is based on the laws of physics. A neural network is a concept to duplicate the work function of the human brain. In this study, the archimedes optimization algorithm (AOA) will be proposed to improve the performance of the feed forward neural network (FFNN). This hybrid method is called AOA-NN. The hybrid method is used to improve the performance of power system stabilizers. To test the ability and effectiveness of the AOA-NN method, a comparison with the conventional PSS, feed-forward neural network (FFNN), Cascade-forward neural network (CFNN), Distributed time-delay neural network (DTDNN) and Sine Tree-Seed Algorithm - Feed-forward Neural network (STSA-NN) method are applied. From the research, it can be concluded that the method proposed by AOA-NN has the best ability. The AOA-NN method has the ability to reduce the overshoot speed with an average value of $85.43972 \%$ and the overshoot rotor angle with an average value of $38.9278 \%$.
\end{abstract}

Keywords: Archimedes optimization algorithm, Feed-forward neural network, Power system stabilizer, Nature inspired algorithms, Metaheuristic.

\section{Introduction}

Keeping the power system in a stable condition is a challenge and difficult in the future. This is influenced by a variety of loads and networks that are increasing sharply [1]. When a system is in trouble, the generator will experience wobbling and lose synchronization. This has a major effect on system dynamics. The power system is a system of compound and character. It is influenced by complexity and multi-components. The system has its own behavior. This is due to different loads. In addition, the generator has its own scheme or schedule.

The application of an automatic voltage regulator (AVR) with a high gain value in the excitation system at the generator is created new problems. This results in low frequency electromechanical oscillations can reduce the damping torque. The application of an automatic voltage regulator (AVR) with a high gain value in the excitation system at the generator creates its own problems. This is resulting in low-frequency electromechanical oscillations having an effect by reducing the damping torque. Low frequency oscillations are frequent and very detrimental [2]. Because it will affect the maximum power transfer and power system safety

To fix this problem, the power system stabilizer (PSS) has function as a stabilizing signal to the automatic voltage regulator (AVR). The excitation of the generator will be modulated by the PSS to reduce the electric torque component with deviation of the rotor speed. This is to increase the damping of the generator. Many power companies are using PSS because it has the character of having a simple structure, easy to implement and flexible. PSS performance will experience changes due to nonconstant operation of the power system. The popular 
PSS is applied based on classical linear control modeling [3] namely conventional PSS (CPSS). This modeling has constant parameters and power system settings. This modeling is suitable for use in a system that is constant and slightly changes [4]. For setting parameters on a large-scale power system which is collected from several single machines is not an easy thing. When one system has a good damping value. On the other hand, some systems are not damped. This is exacerbated by the ever-changing load.

The development of computational theory and algorithms has prompted researchers to focus switching conventional PSS to intelligent control based on artificial intelligence. In recent years, research using artificial intelligence has been widely presented.

Cuckoo search optimization (CSO) is a method based on the cuckoo bird. This is an attractive bird that has a nice voice. On the other hand, they are aggressive reproductive strategies. PSS research uses the Cuckoo search optimization method as presented by Chitara et al [5]. In the paper, the CSO method is explored for the optimal and robust power system stabilizer (PSS) design for multi engine power systems. Testing using the New England 10-engine, 39-bus Power System. A CSO based study also conducted by Djalal et al [6] investigated the placement and adjustment of PSS under N-1 contingency. A study presented by Verma et al [7] who designed PID-PSS with the help of the CSO method. This study uses a single machine in testing. Bat algorithm (BA) is a bio-inspired algorithm. The bat algorithm is based on the echolocation or biosonar characteristics of the microbats. Research that discusses BA as applied to PSS as presented by Chaib et al [8]. The paper is used the BA method in the hybridization of a fractional order PID controller (pikdl) and PSS for optimal stabilizer (FOPID-PSS). This study uses a single machine. A BA-based study was also presented by Djalal et al [9] who applied PSS to a $150 \mathrm{kv}$ Sulselrabar system. Increasing the ability of the BA method as presented by Baadji et all [10], namely the Comprehensive Learning Bat Algorithm applied to the coordination of Power System Stabilizers (PSS) and Static Var Compensator (SVC). The paper is used a $400 \mathrm{MW}$ multimachine system.

The whale optimization algorithm (WOA) is based on this unique whale hunting method. This method observes the foraging method of humpback whales. This whale has a very special hunting method. Its name is called bubble net feeding. The whale creates two paths to reach its prey. Dasu et al [11] applied the WOA method to PSS using a multimachine system consisting of 3 generators and nine buses. The research conducted performance measurement by comparing the Particle swarm optimization (PSO) and Differential evolution (DE) methods. The application of WOA to PSS was also presented by Sahu et al [12]. The improved capability of the WOA method was presented by Sahu et al [13]. This research modifies the WOA for a coordinated structure that combines a static synchronous series compensator and a power system stabilizer. This method is called a modified whale optimization algorithm (MWOA).

The Salp Swarm Algorithm (SSA) method is an algorithm that mimics the navigating and foraging behavior of salp fish in the oceans. Ekinci et al [14] applied the SSA method to the PSS tested on 3 engines, 9 bus power systems. Performance comparison used the Tabu Search (TS) and the Biogeography-Based Optimization (BBO) methods. Bacterial foraging optimization (BFO) is based on social foraging behavior. Escherichia Coli (E. Coli) bacteria simulate the search for food in the human intestine. Ibrahim et al [15] designed a BFO-based PSS that was applied to a multimachine 4 machines 11-buses 2-areas power system. In this study, the proposed BFO-based PSS was compared with three PSS, namely the simplified multi-band (MB) PSS, Conventional Delta PSS from P. Kundur, and the Conventional Acceleration Power (Delta Pa) PSS. Research by Ray et al [16] presented the optimization of PSS with BFO and PSO for increased system stability in the SMIB system. The innovation of the BFO method by combining it with other methods such as Fuzzy is presented by Khorram et al [17]. This method is called Fuzzy Adaptive Bacterial Foraging (FABF). This study presents a PSS design based on Fuzzy Adaptive Bacterial Foraging (FABF) which is tested on a 10-engine multimachine system, 39-bus New England Power System.

The Firefly Algorithm (FA) method is based on the behavior of fireflies and their blinking patterns. Several FA-based studies applied to PSS are widely presented. Farhad et al [18] presented a single machine system. This study presents a performance comparison with the BA method.

Some researchers present PSS based on fuzzy methods. Shokouhandeh et al [19] presented an optimized and innovative type-2 fuzzy PID power system stabilizer (PSS). This stabilizer is a combination of a PID controller and a type-2 fuzzy set whose parameters have been optimized using the Hybrid Big Bang - Big Crunch (HBB - BC) algorithm. Khaddouj et al [20] presented a slidding mode controller (SMC) design combined with a type-2 fuzzy power system stabilizer (PSS). Testing using a single machine system. 
The use of the neural network method that is applied to PSS is also not little. Masrob et al [21] present a simple artificial neural network power system stabilizer. testing using a single machine system. The use of one of the neural network methods, namely the Distributed Time-Delay Neural Network, is presented by Aribowo [22]. The performance comparison in the paper uses the Recurrent Neural Network PSS (RNN-PSS) and Conventional PSS (CPSS) methods. The application of the Cascadeforward neural network algorithm on PSS is presented by Aribowo et al [23]. The single machine system is used to test PSS performance.

The Archimedes optimization algorithm is presented by Hashim et al in 2020 [24]. Algorithm Archimedes optimization algorithm is a metaheuristic method based on population. Neural networks are one of the most popular artificial intelligence and are often applied in various fields.

This paper will explore the potential of metaheuristic methods, namely, Archimedes optimization algorithm (AOA) [24] to solve controlling parameter of PSS combined with feed forward neural network (FFNN). FFNN method is the most popular method used in various kinds of problem solving in engineering. FFNN has the flexibility and complexity of predicting nonlinear functions to the desired accuracy by varying the number of layers and hidden neurons in each of the layers respectively. The system is tested with 3 test scenarios. The contribution of this paper is

1. The application of the latest and promising metaheuristic methods namely Archimedes optimization algorithm to combine with NN. The Archimedes optimization algorithm method is used to improve the ability of the neural network through weighting.

2. Tested the method used in 3 case studies and comparison with other methods. Effectiveness and reliability testing of the proposed method is compared to CPSS, FFNN, DTDNN [22], CFNN [23], and STSA-NN.

The paper is organized as follows: the second part provides a complete study of a brief description of the metaheuristic methods used in this paper, neural network and power system stabilizer. Section 3 presents the results and performance comparison analysis. In the last section, a conclusion is drawn.

\section{Literature review}

\subsection{An archimedes optimization algorithm}

The Archimedes optimization algorithm (AOA) is a metaheuristic method that is conceptualized from

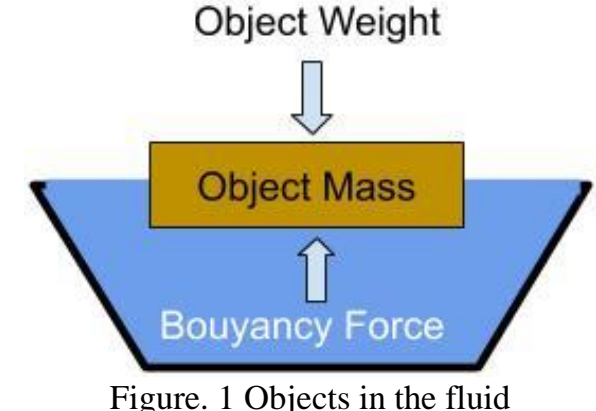

an interesting physical law, namely the Archimedes Principle. The archimede concept declares when an object is submerged either partially or completely in a fluid. The fluid will labor an upward force on the object. The force is common to the weight of the fluid displaced by the object. It is named buoyant force. Fig. 1 is displayed when an object is plunged in a fluid. AOA is maintaining a balance between exploration and exploitation because $\mathrm{AOA}$ is storing multiple solutions and investigates large areas to find the best global solutions [24].

The concept of AOA is like any other metaheuristic method that is population based. AOA starts the algorithm by initializing the population with random speed, density and volume. This will randomize the position in the fluid. Iteration will be carried out after the initial population suitability evaluation and until the conditions fulfilled. The volume and density of the objects will be updated with each iteration. Whereas, the update of the object's acceleration is based on the result of collisions with adjacent objects.

The current position of an object is determined by the updates of its acceleration, density and volume. AOA algorithmically has good global optimization in the exploration and exploitation process. Mathematically, the AOA algorithm can be formulated as follows:

\section{Step 1- Initialization of the position}

$$
X_{i}=l b_{i}+\text { rand } \times\left(u b_{i}-l b_{i}\right) ; i=1,2,3, \ldots . ., M
$$

Where the upper and lower boundaries of the search space are $u b_{i}$ and $l b_{i}$. The ith entity in a population of $n$ entity is $X_{i}$. The formula of the volume ( $\mathrm{vol}$ ) and density (den) is using (2) and (3).

$$
\begin{aligned}
& \operatorname{den}_{i}=\text { rand } \\
& \operatorname{vol}_{i}=\text { rand }
\end{aligned}
$$


Where metrical vector randomly produced values between $[0,1]$ is rand. Evaluation of the initial population and determining the optimal fitness value are carried out in this phase. Evaluation of the initial population and determining the optimal fitness value are carried out in this phase. The optimal values obtained are $d e n_{\text {best }}$, vol $_{\text {best }}, a_{c c}$ best , and $x_{\text {best }}$.

\section{Step 2 - Regenerated volumes and densities}

The volume and density of entity $i$ for the iteration is regenerated applying (4) and (5).

$$
\begin{aligned}
& \operatorname{den}_{i}^{t+1}=\operatorname{den}_{i}^{1}+\operatorname{rand} \times\left(\text { den }_{\text {best }}-\operatorname{den}_{i}^{1}\right)(4) \\
& \operatorname{vol}_{i}^{t+1}=\operatorname{vol}_{i}^{1}+\text { rand } \times\left(\text { vol }_{\text {best }}-\text { vol }_{i}^{1}\right)
\end{aligned}
$$

\section{Step 3 - Set density factor and transfer operator}

An equilibrium state occurs after a collision between objects. The transfer operator functions to change the search mode from exploration to exploitation. This can be formulated as follows:

$$
T F=\exp \left(\frac{t-t_{\max }}{t_{\max }}\right)
$$

Where the $T F$ value increases with a limit of 1 . The maximum iterations and iterations are represented by $t$ and $t_{\text {max }}$. Global to local searches are optimized with density $(d)$ which decreases over time.

$$
d^{t+1}=\exp \left(\frac{t-t_{\max }}{t_{\max }}\right)-\left(\frac{t}{t_{\max }}\right)
$$

Where the ability to reach promising areas that have been discovered is $d^{t+1}$. This will maintain a balance between exploitation and exploration.

\section{Step $4-$ Exploration $(T F \leq 0.5)$ and exploitation (TF $>0.5)$}

The collisions between entities occur when $T F \leq$ 0.5. It is Exploration phase. A random material $\left(d e n_{m r}, v o l_{m r}\right)$ is selected and the object speed is updated in this step. This is used Eq. (8):

$$
a c c_{i}^{t+1}=\frac{d e n_{m r}+v o l_{m r} \times a c c_{m r}}{\operatorname{den}_{i}^{t+1} \times v o l_{i}^{t+1}}
$$

Changes in the value of 0.5 will affect explorationexploitation behavior. A value of $T F \leq 0.5$ will keep the exploration longer. Next, It is a exploitation $(T F>0.5)$. There is no crash between entities.

$$
\operatorname{acc}_{i}^{t+1}=\frac{\text { den }_{\text {best }}+\text { vol }_{\text {best }} \times a c c_{\text {best }}}{\operatorname{den}_{i}^{t+1} \times v o l_{i}^{t+1}}
$$

Where the velocity of the best entity is $a c c_{\text {best }}$

\section{Step 5 - Normalize velocity}

$$
a c c_{i-\text { norm }}^{t+1}=u+\frac{a c c_{i}^{t+1} \times \min (a c c)}{\max (a c c)-\min (a c c)}+l
$$

Where $u$ and $l$ are the space of normalization. It is set to 0.9 and 0.1 . The $a c c_{i-n o r m}^{t+1}$ establishes the possibility of pace that each individual will shift. The acceleration value will be high if entity $i$ is far from global optimal. It can be said that the entity is in the exploration phase. In normal conditions, the acceleration factor will be at the highest position which will decrease according to the time allocation. Search agents will be encouraged to move away from local solutions to global solutions. In some cases, some agents are not optimal in this regard or take more time.

\section{Step 6 - Regenerate position}

If $T F \leq 0.5$ (exploration phase)

$$
\begin{aligned}
x_{i}^{t+1}= & x_{i}^{t}+C_{1} \times \text { rand } \times \operatorname{acc}_{i-\text { norm }}^{t+1} \times d \times \\
& \left(x_{\text {rand }}-x_{i}^{t}\right)
\end{aligned}
$$

If $T F>0.5$ (exploitation phase)

$$
\begin{gathered}
x_{i}^{t+1}=\begin{array}{c}
x_{\text {best }}^{t}+F \times C_{2} \times \text { rand } \\
d \times\left(T \times x_{\text {best }}-x_{i}^{t}\right)
\end{array} \\
T=C_{3} \times T F \\
F=\left\{\begin{array}{l}
+1 \text { if } P \leq 0.5 \\
-1 \text { if } P>0.5
\end{array}\right. \\
P=2 \times \text { rand }-C_{4}+1+\text { norm }
\end{gathered}
$$

Where $C_{1}$ Is constant with a value of 2 and $C_{2}$ Is constant with a value of $6 . T$ is increases with time in space $\left[C_{3} \times 0.3,1\right]$. Initial small percentage value results in a large difference between the current position and the best position. This results in a large random value. Increasing the percentage results in less difference between the current position and the best position. This results in achieving a good balance between exploration and exploitation. $F$ is the notation for changing the aim of motion.

\section{Step 7 - Evaluation}

Each entity will be evaluated using the objective function. the best solutions will be saved with acc $_{\text {best }}$, vol $_{\text {best }}$, den $_{\text {best }}$ and $x_{\text {best }}$. 


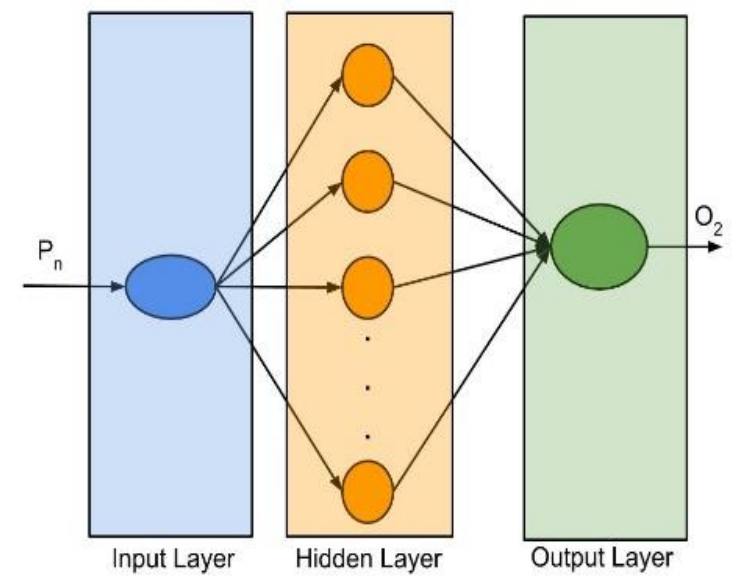

Figure. 2 Topology of neural network

\subsection{Neural network}

Duplicated by the skills from the human brain, neural networks are one of the wrong types of artificial intelligence. ANN simple topology consists of input, hidden and output layers [25]. The topology of neural network can be seen in Fig. 2. Node/neuron is the main item of ANN processing. In the first layer, the input nodes $\left(P_{n}\right)$ admit data from the system outside the ANN. In the second layer, the hidden nodes $\left(O_{1}\right)$ admit data from the first layer. It is for calculation and processing. In the last layer, the output nodes $\left(\mathrm{O}_{2}\right)$ admit data from the second layer and computational of the results. In this paper, the neural network is using feed forward neural network. In some simple cases, it is using one hidden layer can solve the problem. On the other hand, a complex problem is not enough to use one hidden layer.

$$
\begin{aligned}
& O_{1}(t)=\sum_{i=1}^{j} W_{i j} P_{n}(\mathrm{t})+b_{1} \\
& O_{2}(t)=f\left(O_{1}(t)\right)=\frac{1}{1+\exp ^{O_{1}}}
\end{aligned}
$$

\subsection{Power system stabilizer}

In the last few decades, conventional power system stabilizers (PSS) have been very popular in power systems throughout the world. As an additional of control system, PSS is often used as an excitation control system. Conventional PSS are generally designed based on linearisation theory to damp rotor oscillations to the desired operating conditions [26]. This is what is called a stable system The work function of the PSS is to suppress the rotor oscillations in an effort to maintain balance in the system. PSS has been widely applied in single machine and multimachine systems. PSS basically consists of gain, lead-lag, and washout. Washout

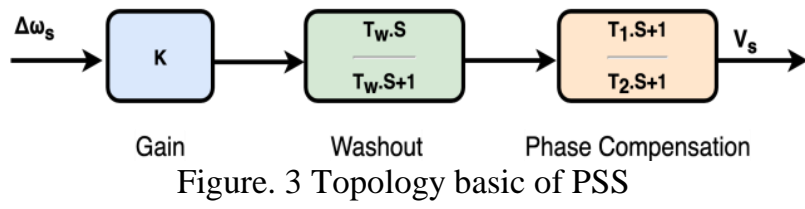

which functions as a high-pass filter that will allow and ensure the passing signal is appropriate and does not change. A lead-lag block or phase compensation that serves as a phase offset. This will supply a phase lead for counterbalance for the lag phase. The Basic structure of PSS can be seen in Fig. 3.

\section{Results and discussion}

As a control for PSS, the topology of the AOANN method is shown in Fig. 4. FFNN method will recognize the incoming data. The best FFNN classification results based on the number of neurons in the hidden layer. The configured neural network will perform data mapping and detection. This will get weighted. The weight obtained is random and will be processed to get the best weight using the AOA method. The optimal population value of AOA is 50 with 100 iterations. Population size and iteration will affect the performance of the AOA-NN method. Lower-bound and upper bound will place the optimal AOA-NN output This process will stop when it reaches the maximum iteration.

Optimization of the neural network algorithm based on the AOA method for PSS control is programmed using MATLAB software. The generator is completed with speed control and turbine, which includes excitation, AVR, and PSS. To prove the robustness of the AOA-NN for the PSS setting, a single-engine power system simulation was performed. Data from conventional PSS are exploited to train and validate using AOA-NN PSS. In this study, it was tested with 3 test scenarios. The topology of the neural network has 5 neurons using the sigmoid activation function. Whereas, the purelin activation function is used in the output layer. In Fig. 5 , PSS based on the AOA-NN method is getting input in the form of speed data from the system output. Meanwhile, the target is learning outcomes using conventional PSS.

The convergence curve of the all methods can be seen in Fig. 6. The proposed method is AOA-NN starting with the first value of 0.9248 . The AOA-NN method has the end value of 0.1355 . On the other hand, the FFNN method has the highest of the first value. It is 1.3080 . The lowest of the first value has DTDNN. The value is 0.1172 . This study uses 3 scenarios to test the effectiveness and durability of 


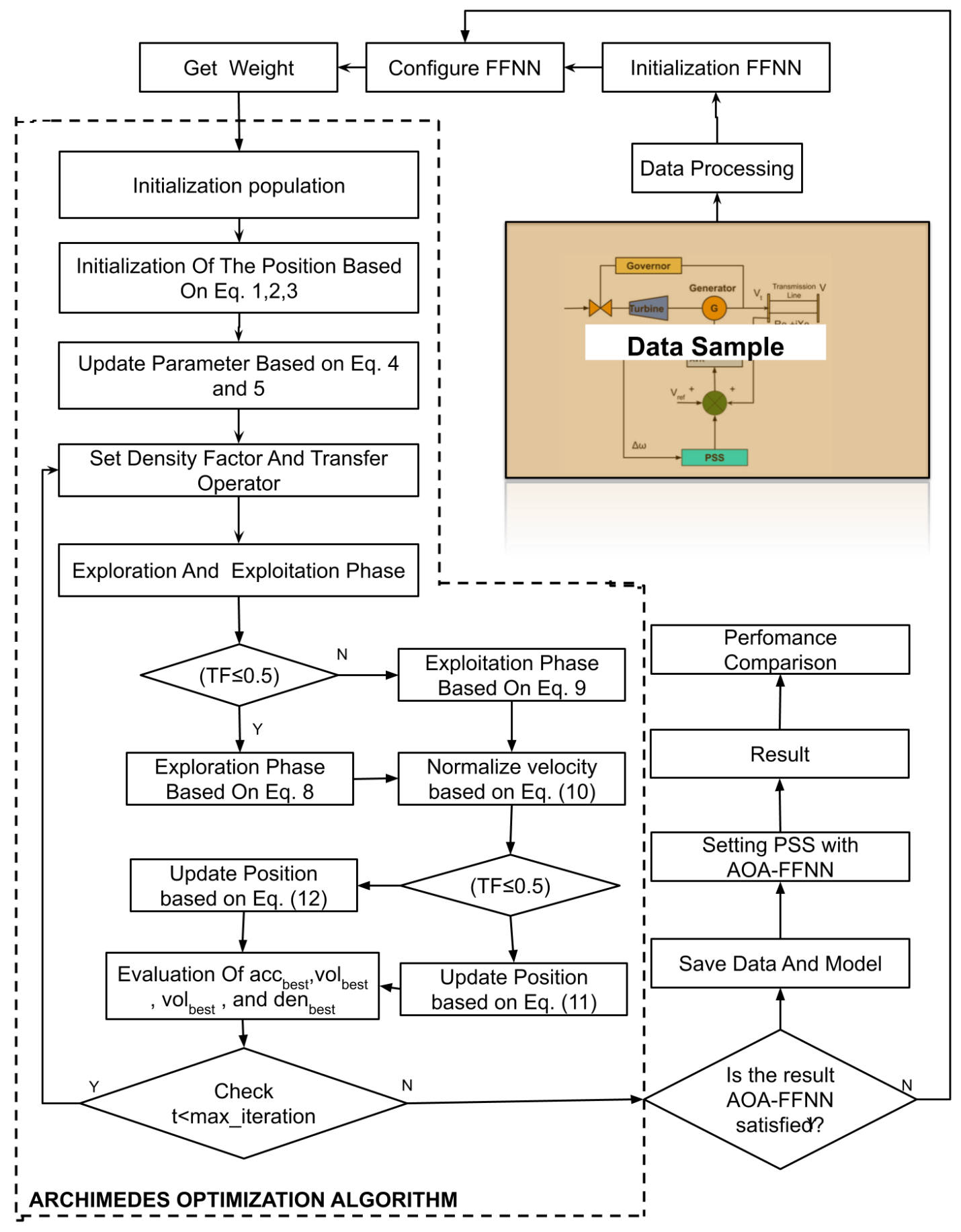

Figure. 4 Diagram of AOA-NN

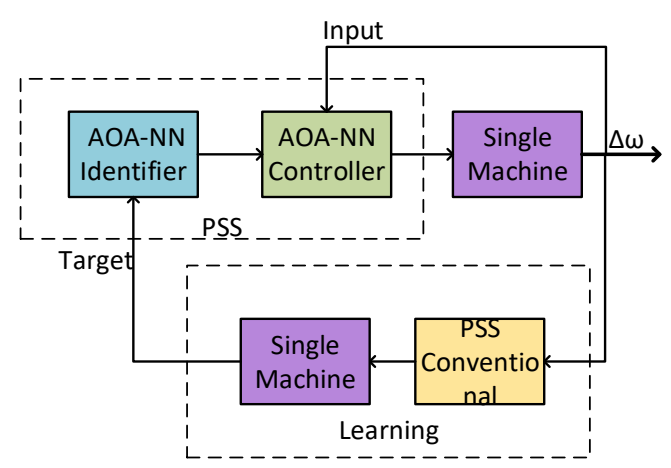

Figure. 5 AOA-NN PSS
Table 1. Parameter of AOA-NN

\begin{tabular}{|c|c|}
\hline Parameter & Value \\
\hline Number Of Population & 50 \\
\hline Maximum Iteration & 100 \\
\hline $\mathrm{C} 1 ; \mathrm{C} 2 ; \mathrm{C} 3 ; \mathrm{C} 4$ & $2 ; 6 ; 2 ; 1$ \\
\hline Lower Bound ; Upper Bound & $-5.12 ; 5.12$ \\
\hline
\end{tabular}

the proposed method. The detail of scenario can be seen in Table 2 


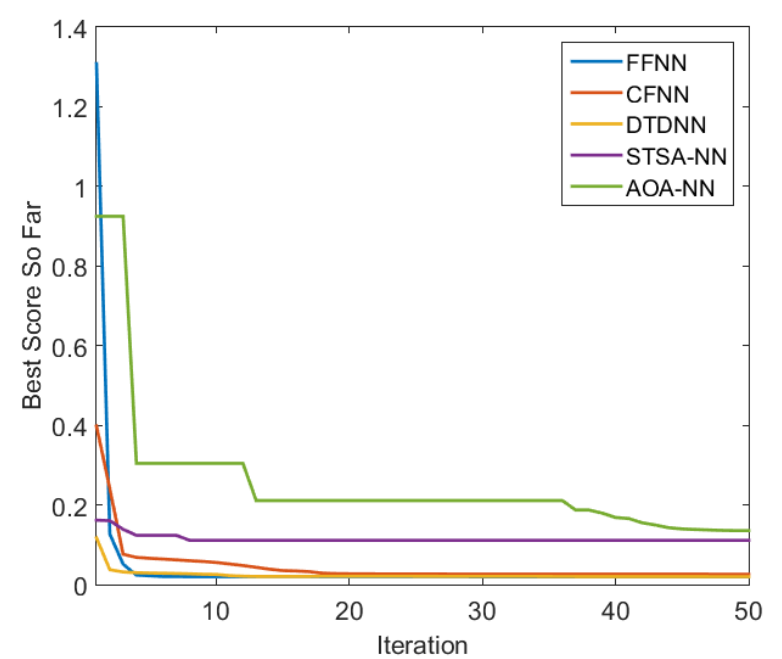

Figure. 6 The convergence curve

Table 2. The scenario of system testing

\begin{tabular}{|c|c|}
\hline Case & Load \\
\hline 1 & Full Load \\
\hline 2 & $50 \%$ of Load \\
\hline 3 & $25 \%$ Of Load \\
\hline
\end{tabular}

In scenario 1 , the single machine system is fully loaded. The response of the system in the form of speed and rotor angle can be seen in Fig. 7. In Fig. 7 (a), the overshoot speed value of the system without PSS is 0.5809. Meanwhile, the overshoot value of the PSS using the AOA-NN method is 0.0858. The proposed method is able to reduce overshoot and undershoot of speed by $85.2298 \%$ and $47.92 \%$. On the other hand, CPSS was only able to reduce overshoot and undershoot of speed by $74.6772 \%$ and

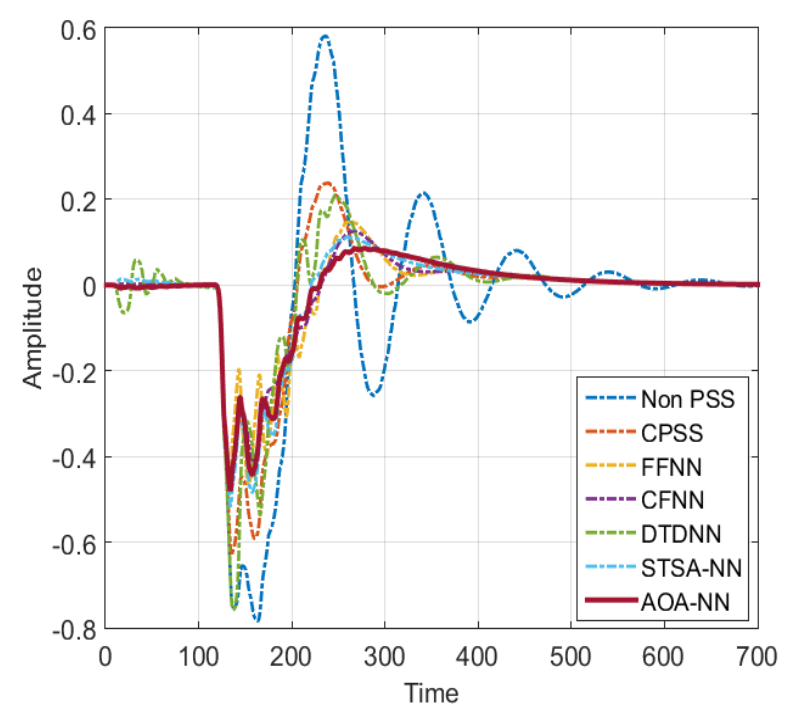

(a)
39.5435\%. In Fig. 7 (b), the undershoot value of the rotor angle of the PSS using the AOA-NN method is -1.611 . The proposed method was able to reduce the undershoot by $38.6052 \%$. This value is slightly below the CFNN method. The undershoot value using CFNN can reduce up to $39.0396 \%$. Table 3 is detail of the result scenario 1 .

The results of scenario 2 can be seen in Fig. 8. Fig. 8 (a) is the speed output. The overshoot speed using the AOA-NN method is 0.0418 and the undershoot is -0.2305 . The application of the AOA-NN method is able to reduce overshoot and undershoot of speed by up to $85.611 \%$ and $41.1539 \%$. The STSA-NN method is slightly less capable than the AOA-NN. The STSA-NN method is able to reduce overshoot and undershoot of speed by up to $81.18704 \%$ and $41.1539 \%$. Meanwhile, the response of the rotor angle for scenario 2 can be seen in Fig. 8 (b). The undershoot value of the proposed method is -1.1976 and the settling time is 568 seconds. The AOA-NN method was able to reduce undershoot by $39.3979 \%$. Application of the CFNN and STSA-NN methods in scenario 2 resulted in an undershoot value of the rotor angle up to $37.2027 \%$ and $37.3018 \%$. The application of CFNN and STSA-NN methods has adjacent values. The detail of Scenario 2 can be seen in Table 4 .

Fig. 9 is the output of scenario 3. The overshoot value of a system without PSS is 0.1453 with a settling time of 473 seconds. The proposed method has an overshoot of speed by 0.0211 with a settling time of 517. In scenario 3, the AOA-NN method is able to reduce overshoot and undershoot speeds by $85.4783 \%$ and $42.4273 \%$. In scenario 3, the DTDNN

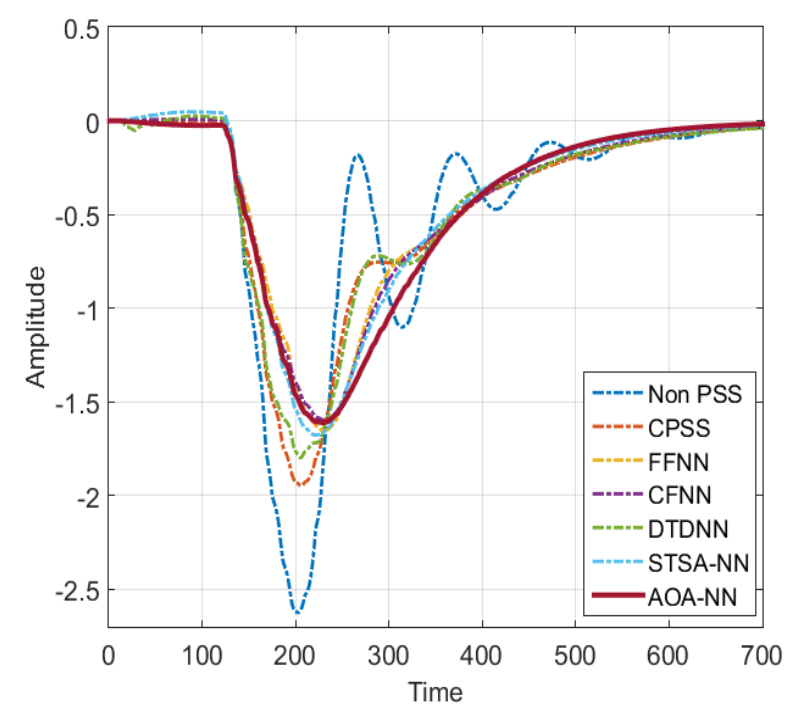

(b)

Figure. 7 Output of scenario 1: (a) speed and (b) rotor angle 
Table 3. The detail of scenario 1

\begin{tabular}{|l|c|c|c|c|c|}
\hline \multirow{2}{*}{ Methods } & \multicolumn{3}{|c|}{ Speed } & \multicolumn{2}{c|}{ Rotor Angle } \\
\cline { 2 - 6 } & Overshoot & Under Shoot & Time Settling & Undershoot & Time Settling \\
\hline Non-PSS & 0.5809 & -0.7842 & 498 & -2.624 & 578 \\
\hline CPSS & 0.2381 & -0.6298 & 430 & -1.943 & 621 \\
\hline FFNN PSS & 0.1471 & -0.4741 & 462.85 & -1.657 & 625 \\
\hline CFNN PSS & 0.1248 & -0.4827 & 519 & -1.5996 & 686 \\
\hline DTDNN PSS & 0.2107 & -0.7572 & 481 & -1.7982 & 684 \\
\hline STSA-NN PSS & 0.1120 & -0.5176 & 502 & -1.6776 & 654 \\
\hline AOA-NN PSS & 0.0858 & -0.4084 & 455 & -1.611 & 571 \\
\hline
\end{tabular}

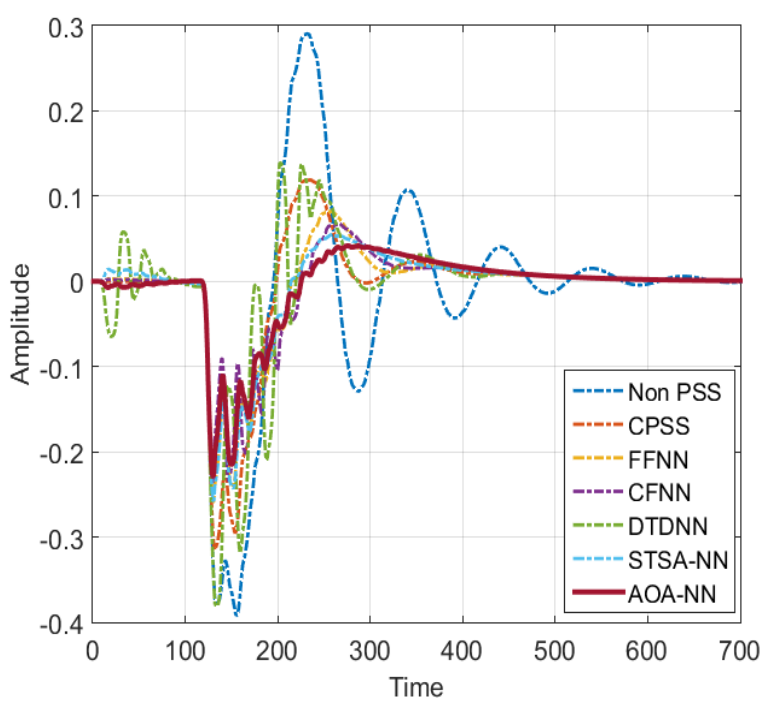

(a)

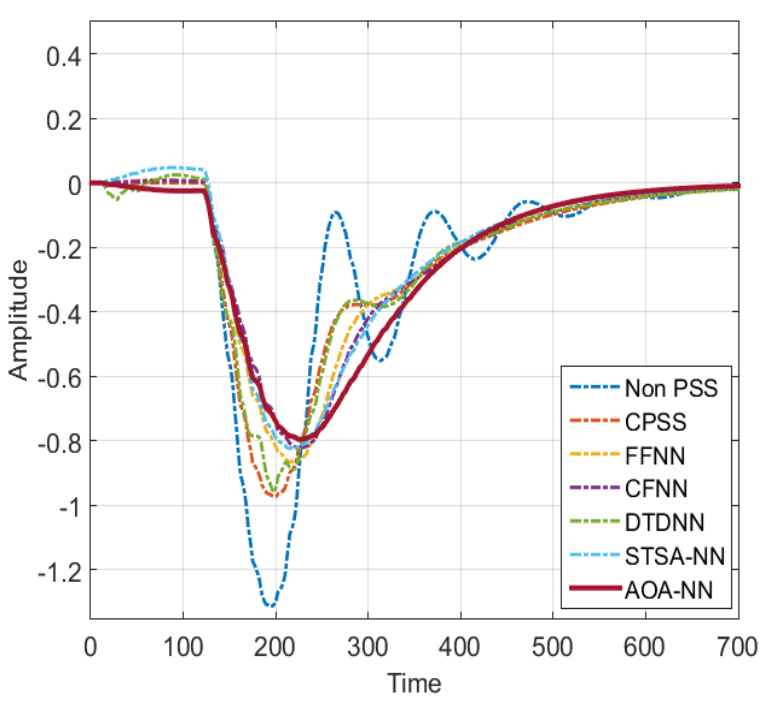

(b)

Figure. 8 Output of scenario 2: (a) speed and (b) rotor angle

Table 4. The detail of scenario 2

\begin{tabular}{|l|c|c|c|c|c|}
\hline \multirow{2}{*}{ Methods } & \multicolumn{3}{|c|}{ Speed } & \multicolumn{2}{c|}{ Rotor Angle } \\
\cline { 2 - 6 } & Overshoot & Under Shoot & Time Settling & Undershoot & Time Settling \\
\hline Non-PSS & 0.2905 & -0.3917 & 599 & -1.3120 & 641 \\
\hline CPSS PSS & 0.1190 & -0.3149 & 489 & -0.9736 & 684 \\
\hline FFNN PSS & 0.0821 & -0.2590 & 509 & -0.8645 & 680 \\
\hline CFNN PSS & 0.0675 & -0.2359 & 521 & -0.8239 & 682 \\
\hline DTDNN PSS & 0.1389 & -0.3813 & 478 & -0.9598 & 675 \\
\hline STSA-NN PSS & 0.0547 & -0.2573 & 501 & -0.8226 & 655 \\
\hline AOA-NN PSS & 0.0418 & -0.2305 & 522 & -0.7951 & 638 \\
\hline
\end{tabular}

method experienced a fairly high decrease in performance. In the DTDNN method, the overshoot speed is only able to reduce by $3.0282 \%$. while the speed undershoot value failed, adding up to $14.9924 \%$ oscillation. The value of overshoot and undershoot speed is close to the value of the AOANN method, namely the STSA-NN method. the values are $81.6242 \%$ and $33.5033 \%$. Meanwhile, the undershoot of rotor angle is -0.4016 with a settling time of 630 . In scenario 3, the application of the
AOA-NN method has the ability to reduce the undershoot rotor angle slightly carried by STSA-NN. the application of STSA-NN was able to reduce the undershoot rotor angle by $39.1768 \%$. while the AOA$\mathrm{NN}$ method has the ability to reduce undershoot rotor angle by $38.7805 \%$. The difference between the STSA-NN and AOA-NN methods in reducing undershoot rotor angle is 0.0026 or $0.3963 \%$. The detail of Scenario 3 can be seen in Table 5 . 
Table 5. The detail of scenario 3

\begin{tabular}{|l|c|c|c|c|c|}
\hline \multirow{2}{*}{ Methods } & \multicolumn{3}{|c|}{ Speed } & \multicolumn{2}{c|}{ Rotor Angle } \\
\cline { 2 - 6 } & Overshoot & Under Shoot & Time Settling & Undershoot & Time Settling \\
\hline Non-PSS & 0.1453 & -0.1961 & 550 & -0.6560 & 632 \\
\hline CPSS & 0.0595 & -0.1580 & 480 & -0.4868 & 675 \\
\hline FFNN PSS & 0.0479 & -0.1389 & 489 & -0.4588 & 661 \\
\hline CFNN PSS & 0.0386 & -0.1318 & 492 & -0.4398 & 661 \\
\hline DTDNN PSS & 0.1409 & -0.2255 & 445 & -0.5035 & 661 \\
\hline STSA-NN PSS & 0.0267 & -0.1304 & 488 & -0.3990 & 646 \\
\hline AOA-NN PSS & 0.0211 & -0.1129 & 517 & -0.4016 & 630 \\
\hline
\end{tabular}

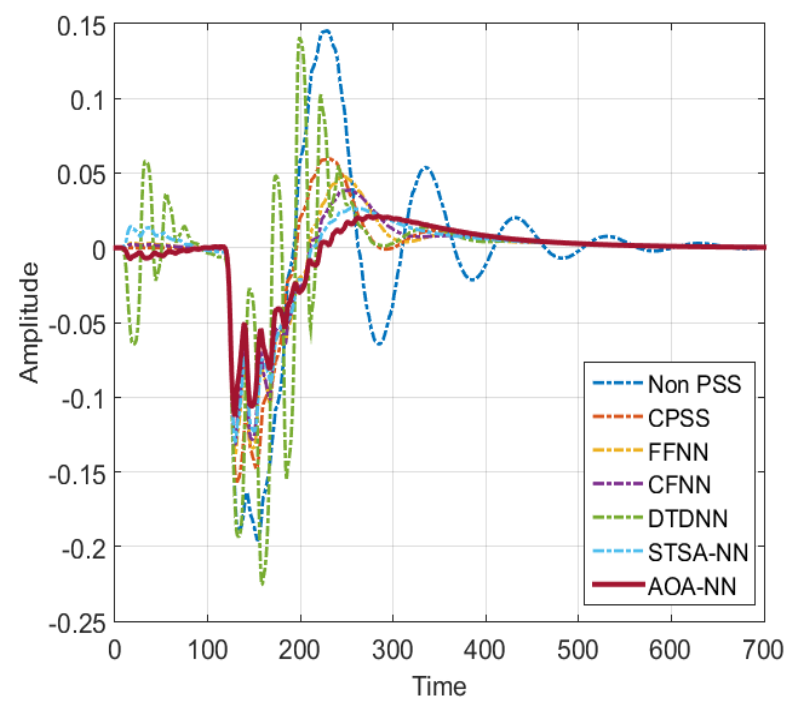

(a)

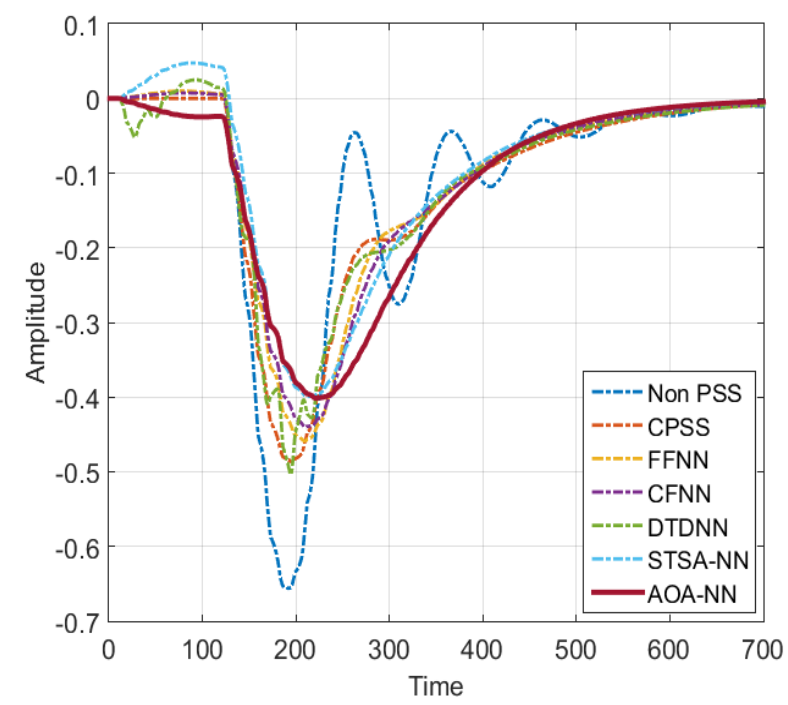

(b)

Figure. 9 Output of scenario 3 (a) speed and (b) lotor angle

\section{Conclusion}

The research is to propose a hybrid AOA-NN method to improve the performance of power system stabilizers. To improve the performance of power system stabilizers, learning and training methods are used using data from the system. The data from the system taken is the output data in the form of speed. This data consists of data from systems with and without conventional PSS. This study uses 3 scenarios in testing the effectiveness and toughness of the proposed system. The proposed method has the benefit of increasing the performance of the FFNN. This can be seen from the results of scenario 1 to scenario 3. In scenario 1, the use of the AOA-NN method can reduce overshoot and undershoot by $41.67 \%$ and $13.857 \%$ from the speed compared to FFNN PSS. Meanwhile, the rotor angle can be reduced by $2.77 \%$. In scenario 2, AOA-NN can reduce overshoot and undershoot by $49.08 \%$ and $11 \%$ of the speed compared to FFNN PSS. The undershoot of the rotor angle in scenario 2 can be reduced by $8.03 \%$ compared to the FFNN PSS. Finally, the AOA-NN method can reduce the overshoot and undershoot values by $55.95 \%$ and $18.72 \%$. In scenario 3 , the rotor angle is reduced by $12.47 \%$ compared to the FFNN PSS. By giving the addition of the AOA method to the FFNN, the oscillation damping speed and rotor angle are increased. In this study, the proposed method AOANN was tested on a simple system. In order to determine the robustness and reliability of the proposed method, it is necessary to carry out test cases on a more complex and non-linear system.

\section{Conflicts of Interest}

The authors declare no conflict of interest This article has not been published and is not under consideration for publication elsewhere. The authors have no conflict of interest in regard to this research or its funding. 


\section{Author Contributions}

The first author, Widi Aribowo is contributed to the implementation of algorithms, the conduct of experiments, and the formation of the paper. Supari Muslim and Bambang Suprianto are contributed to supervision and checking the paper. Subuh Isnur Haryudo and Aditya Chandra Hermawan are contributed in resource data and analysis data.

\section{References}

[1] B. Douidi, L. Mokrani, and M. Machmoum, "A New Cascade Fuzzy Power System Stabilizer for Multi-machine System Stability Enhancement", J. Control Autom. Electr. Syst., Vol. 30, pp. 765-779, 2019.

[2] J. Qi, Q. Wu, Y. Zhang, G. Weng, and D. Zhou, "Unified Residue Method for Design of Compact Wide-area Damping Controller Based on Power System Stabilizer", Journal of Modern Power Systems and Clean Energy, Vol. 8, No. 2, pp. 367-376, 2020.

[3] K. Eltag, M. S. Aslamx, and R. Ullah, "Dynamic Stability Enhancement Using Fuzzy PID Control Technology for Power System", Int. J. Control Autom. Syst., Vol. 17, No. 1, pp. 234242, 2019.

[4] A. Jamal, S. Suripto, and R. Syahputra, "Multi Band Power System Stabilizer Model for Power Flow Optimization in Order to Improve Power System Stability", Journal of Theoretical and Applied Information Technology, Vol. 81, No. 1, pp. 116-123, 2015.

[5] D. Chitara, K. R. Niazi, A. Swarnkar, and N. Gupta, "Cuckoo Search Optimization Algorithm for Designing of a Multimachine Power System Stabilizer", IEEE Transactions on Industry Applications, Vol. 54, No. 4, pp. 3056-3065, 2018.

[6] M. R. Djalal, M. Y. Yunus, H. Setiadi, and A.U. Krismanto, "Small-Signal-Stability Enhancement using a Power-System Stabilizer based on the Cuckoo-Search Algorithm against Contingency N-1 in the Sulselrabar 150-kV System", Makara Journal of Technology, Vol. 22, No. 1, pp. 1-8, 2018.

[7] Sheshnarayan, B. Verma, and P. K. Padhy, "Design PID Controller based PSS using Cuckoo Search Optimization Technique", In: Proc. of 2019 4th International Conf. on Recent Trends on Electronics, Information, Communication \& Technology (RTEICT), Bangalore, India, pp. 459-463, 2019.

[8] L. Chaib, A. Choucha, and S. Arif, "Optimal Design and Tuning of Novel Fractional Order
PID Power System Stabilizer using a New Metaheuristic Bat Algorithm", Ain Shams Engineering Journal, Vol. 8, No. 2, pp. 113-125, 2017.

[9] M. R Djalal, M. Y. Yunus, H. Nawir, and A. Imran, "Application of Smart Bats Algorithm for Optimal Design of Power Stabilizer System at Sengkang Power Plant", International Journal of Artificial Intelligence Research, Vol. 1, No.1, pp. 1-5, 2017.

[10] B. Baadji, H. Bentarzi, and A. Bakdi, "Comprehensive Learning Bat Algorithm for Optimal Coordinated Tuning of Power System Stabilizers and Static VAR Compensator in Power Systems", Engineering Optimization, Vol. 52, No. 10, pp. 1761-1779, 2020.

[11] B. Dasu, M. Sivakumar, and R. Srinivasarao, "Interconnected Multi-Machine Power System Stabilizer Design using Whale Optimization Algorithm", Prot. Control Mod Power Syst., Vol. 4, No. 2, pp. 1-11, 2019.

[12] P. R. Sahu, P. K. Hota, and S. Panda, "Comparison of Grasshopper and Whale Optimization Algorithm for Design of FACTS Controller with Power System Stabilizer", In: Proc. of 2018 Fifth International Conf. on Parallel, Distributed and Grid Computing (PDGC), Solan Himachal Pradesh, India, pp. 424-429, 2018.

[13] P. R. Sahu, P. K. Hota, and S. Panda, "Modified Whale Optimization Algorithm for Coordinated Design of Fuzzy Lead-Lag Structure Based SSSC Controller and Power System Stabilizer", Int. Trans. Electr. Energ. Syst., Vol. 29, No. 4, 2019.

[14] S. Ekinci and B. Hekimoglu, "Parameter optimization of power system stabilizer via Salp Swarm algorithm", In: Proc. of 2018 5th International Conf. on Electrical and Electronic Engineering (ICEEE), Istanbul, pp. 143-147, 2018.

[15] N. M. A. Ibrahim, B. E. Elnaghi, H. A. Ibrahim, and H. E. A. Talaat, "Performance Assessment of Bacterial Foraging Based Power System Stabilizer in Multi-Machine Power System", International Journal of Intelligent Systems and Applications, Vol. 11, No. 7, pp. 43-53, 2019.

[16] P. K. Ray, S. R. Paital, A. Mohanty, T. K. Panigrahi, M. Kumar, and H. Dubey, "Swarm and bacterial foraging based optimal power system stabilizer for stability improvement", In: Proc. of 2016 IEEE Region 10 Conf. (TENCON), Singapore, pp. 1916-1920, 2016.

[17] B. Khorram and H. Lesani, "Coordinated Control of Flexible AC Transmission System Devices and Power System Stabilizer for 
improve the Power System Stability Using

Fuzzy Adaptive Bacterial Foraging", International Journal on Electrical Engineering and Informatics, Vol. 7, No. 2, pp. 334-351, 2015.

[18] Z. Farhad, I. Eke, S. S. Tezcan, and S. J. Safi, “A Robust PID Power System Stabilizer Design of Single Machine Infinite Bus System using Firefly Algorithm", Gazi University Journal of Science, Vol. 31, pp. 155-172, 2018.

[19] H. Shokouhandeh and M. Jazaeri, "An Enhanced and Auto Tuned Power System Stabilizer Based On Optimized Interval Type-2 Fuzzy PID Scheme", Int. Trans. Electr. Energ. Syst., Vol. 28, No. 1, 2018.

[20] B. M. khaddouj, D. Faiza, and I. Boumhidi, "Design of Type-2 Fuzzy PSS combined with Sliding Mode Control for Power System SMIB", In: Proc. of 2020 1st International Conf. on Innovative Research in Applied Science, Engineering and Technology (IRASET), Meknes, Morocco, pp. 1-6, 2020.

[21] M. A. Masrob, M. A. Rahman, and G. H. George, "Design of A Neural Network based Power System Stabilizer in Reduced Order Power System", In: Proc. of 2017 IEEE 30th Canadian Conf. on Electrical and Computer Engineering (CCECE), Windsor, ON, pp. 1-6, 2017.

[22] W. Aribowo, "Tuning for Power System Stabilizer Using Distributed Time-Delay Neural Network", SINERGI, Vol. 22, No. 3, pp. 205210, 2017.

[23] W. Aribowo, S. Muslim, munoto, B. Suprianto, U. T. Kartini and I. G. P. Asto Buditjahjanto, "Tuning of Power System Stabilizer Using Cascade Forward Backpropagation", In: Proc. of 2020 Third International Conf. on Vocational Education and Electrical Engineering (ICVEE), Surabaya, Indonesia, pp. 1-5, 2020.

[24] F. A. Hashim, K. Hussain, E. H. Houssein, M. S. Mabrouk, and W. Al-Atabany, "Archimedes Optimization Algorithm: A New Metaheuristic Algorithm for Solving Optimization Problems", Appl. Intell., 2020.

[25] L. D. Medus, T. Iakymchuk, J. V. FrancesVillora, M. Bataller-Mompeán, and A. RosadoMuñoz, "A Novel Systolic Parallel Hardware Architecture for the FPGA Acceleration of Feed-forward Neural Networks", IEEE Access, Vol. 7, pp. 76084-76103, 2019.

[26] R. Ramya and S. Maity, "Enhancement of Small Signal Stability of SMIB System with Robust Power System Stabilizer", Int. J. Recent Technol. Eng., Vol. 8, No. 2S11, pp. 3416-3423, 2019. 